\title{
The transport disruption ontology.
}

CORSAR, D., MARKOVIC, M., EDWARDS, P. and NELSON, J.D. 


\title{
The Transport Disruption Ontology
}

\author{
David Corsar, Milan Markovic, Peter Edwards, and John D. Nelson \\ dot.rural Digital Economy Hub, University of Aberdeen, Aberdeen, AB24 5UA \\ \{dcorsar,m.markovic,p.edwards,j.d.nelson\}@abdn.ac.uk
}

\begin{abstract}
This paper presents the Transport Disruption ontology, a formal framework for modelling travel and transport related events that have a disruptive impact on traveller's journeys. We discuss related models, describe how transport events and their impacts are captured, and outline use of the ontology within an interlinked repository of the travel information to support intelligent transport systems.
\end{abstract}

\section{Introduction}

Transport is frequently viewed as a key sector within smart cities for improving citizen's quality of life [2], [8], [13]. Such visions anticipate that IT systems will utilise data made available by the integration of physical and digital transport infrastructures to address the mobility challenges faced by cities today. One such challenge is minimising the impact of transport disruptions [4]: road congestion is estimated to cost an average of 1\% of GDP across the European Union [4], while the absence of real-time information about the impact of disruptions is a major factor in the dissatisfaction with, and reduced attractiveness of public transport $[10,11]$. However, tackling such problems requires addressing the challenges of data interoperability, analysis, information extraction, and reasoning presented by such environments - challenges that Semantic Web and linked-data are key technologies in overcoming [9].

The shared models and vocabularies provided by ontologies are fundamental to any Semantic Web solution. Ontologies have previously been used to create an integrated ecosystem of the transport information required to support a real-time passenger information system [3]. The Transport Disruption ontology described in this paper enables the extension of such ecosystems with details of travel and transport related events that can have disruptive impacts on mobility. As such, the Transport Disruption ontology contributes a key model to the ongoing work of the Semantic Web community in addressing the data challenges of smart cities, and a major transport challenge faced by society.

This paper is structured as follows: section 2 describes the ontology; section 3 details its application in a use case; section 4 discusses related work; and section 5 concludes the paper.

* The research described here is supported by the award made by the RCUK Digital Economy programme to the dot.rural Digital Economy Hub; award reference: $\mathrm{EP} / \mathrm{G} 066051 / 1$ 


\section{The Transport Disruption Ontology}

The Transport Disruption ontology provides a formal framework for modelling travel and transport related events that can have a disruptive impact on an agent's planned travel. The ontology is available at its namespace:

http: //purl.org/td/transportdisruption\#, abbreviated to 'td' throughout, and the associated github project ${ }^{1}$. Figure 1 outlines the ontology schema.

The ontology was defined following an analysis of disruption information provided by transport authorities and operators of bus, rail, ferry, and air public transport services in the UK. This identified the requirement to capture the occurence of an event in terms of its type, location, time period, compound and causal relationships to other events, and any impact experienced by agent(s) that have to adapt their plan(s) because of it. Following linked-data publishing best practise [5], Linked Open Vocabularies ${ }^{2}$ and the Linked Open Data ${ }^{3}$ cloud were reviewed to identify existing ontologies that could be used to meet these requirements. The selected ontologies and their integration with the Transport Disruption ontology are discussed below.

The main modelling choice focused on the representation of disruptions. The transport research community [6,7], [12] define the notion of disruptive events, i.e. events that "impact on the supply of transport (infrastructure or services), costs of using transport, or some combination" [7]. Such events can affect single or multiple transport links in a given area, and impact on travellers in a way greater than that experienced through the typical day-to-day variability in travel plans.

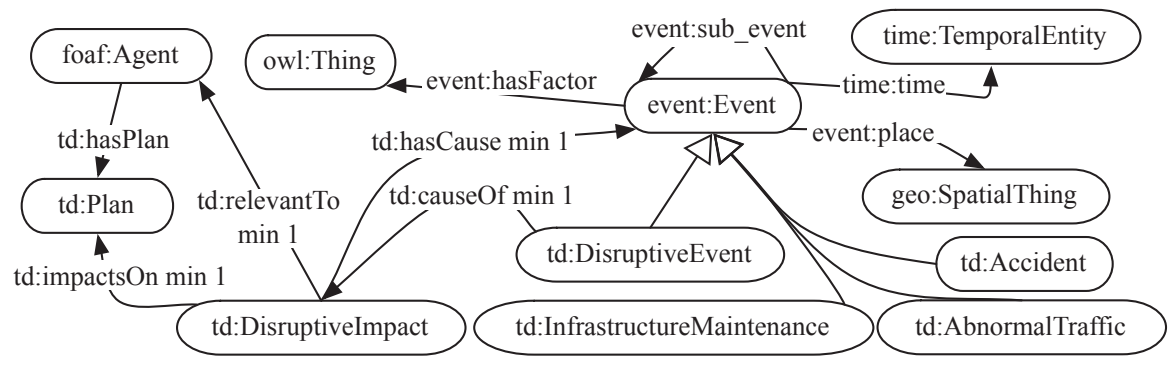

Fig. 1. The Transport Disruption ontology schema, with example transport events.

This is captured by the $t d$ :DisruptiveImpact and $t d$ :DisruptiveEvent classes in the Transport Disruption ontology. A disruptive impact is defined as the

\footnotetext{
1 https://github.com/transportdisruption/transportdisruption.github.io

2 http://lov.okfn.org/dataset/lov

3 http://lod-cloud.net
} 
action of one or more events causing or tending to prevent an agent's planned travel from continuing as expected. The $t d$ :DisruptiveEvent class is aligned to the Event ontology ${ }^{4}$ as a subclass of event:Event. $t$ d:DisruptiveEvent is designed to allow events of any type that have caused a $t$ d:DisruptiveImpact to be classified as disruptive; as such, it is not the intention that this class is extended further. The Event ontology defines events as an arbitrary classification of a space/time region, by a cognitive agent. This aligns with the transport research perspective of disruptive events as, for example, a traveller classifying roadworks occurring within an area and time that they are traveling as causing an undesirable impact on their journey. Use of the Event ontology also allows existing data defined with this model to be used with the Transport Disruption ontology.

$t d$ :DisruptiveImpact is an extension point for developers to define the types of impact relevant to their intended usage of the ontology. This may include descriptions of delays to an agent's journey or details of a journey that cannot be completed due to, for example, a cancelled flight. A minimum cardinality constraint defines that each $t d$ :DisruptiveImpact individual must define the foaf:Agent that it is $t d$ :relevantTo. The Friend-of-a-Friend (FOAF) ontology ${ }^{5}$ was selected as it is a commonly used model for describing people, organisations, and groups, and its use allows reuse of existing FOAF data with the Transport Disruption ontology.

$t d$ :Plan provides an extension point for developers to describe an agent's plan that may be impacted by an event. For example, this could be a person's travel itinerary defined by an expected departure time, location and means of transport to a destination; or a bus schedule providing expected arrival times at stops along a route as defined by the bus operator. Each $t d$ :DisruptiveImpact also references at least one $t d$ :Plan that it $t d: i m p a c t s O n$.

A cardinality constraint is defined stating that each individual of type $t d: D i s$ ruptiveImpact must reference at least one event:Event through the td:hasCause property. Similarly, each $t d$ :DisruptiveEvent must reference at least one $t d$ :DisruptiveImpact that it caused via the $t$ d:causeOf property. An owl:inverse Of relationship is defined between $t$ d:hasCause and $t d$ :cause $O f$, ensuring the correspondences between individuals of these classes can be materialised by a description logic reasoner. A reasoner can then also infer the $t d$ :DisruptiveEvent type for event:Events that have caused a $t d$ :DisruptiveImpact.

New concepts extending event:Event are defined to capture the different types of events that may disrupt an agent's travel plans. These are based on the analysis of published disruption information and aspects of the DATEX $\mathrm{II}^{6}$ specification. DATEX II models the road traffic domain and exchange of data between road management systems, including types of road disruption. The types of events modelled as subclasses of event:Event include $t d$ :OperatorAction, $t d$ :InfrastructureManagement, $t d$ :RoadClosure, $t d$ :SpeedRestriction, $t d$ :Infrastructure Construction, $t d$ :InfrastructureMaintenance, $t d$ :TrafficElementEvent, $t d: A b$ normalTraffic, $t d$ :Accident, $t d$ :EnvironmentalConditions, $t d$ :InfrastructureFailure,

\footnotetext{
${ }^{4}$ Namespace http://purl.org/NET/c4dm/event.owl\#, abbreviated to 'event'.

${ }^{5}$ Namespace http://xmlns.com/foaf/0.1/, abbreviated to 'foaf'.

${ }^{6}$ http://www.datex2.eu/
} 
td:InfrastructureFault, td:Obstruction, $t d: P u b l i c$ TransportEvent, $t d: P u b l i c T r a n s-$ portDelay, td:PublicTransportCancellation, and td:PublicTransportDiversion.

The Event ontology defines that events are associated with a temporal region describing their time of occurrence using the OWL-Time ontology ${ }^{7}$. The Timeline ontology ${ }^{8}$ is also recommended to model intervals in terms of start and end timestamps. Defining such temporal intervals for transport-related events is encouraged as it can be used to determine if an event is completed, active, or planned, which are frequently used terms for defining the status of disruptions.

The Event ontology also defines that events are associated with a spatial object that locates the event using the WGS84 ontology ${ }^{9}$. Providers of travel disruption information typically use a variety of location references, including a point with latitude and longitude values, roads, public transport access points (bus stops, railway stations, etc.), and geographic areas such as counties, cities, and localities. Classes from other ontologies representing such locations can be used with the Transport Disruption ontology by defining alignments to the WGS84 ontology. For example, defining the Road class from the LinkedGeoData ontology ${ }^{10}$ or Stop class from the Transit ontology ${ }^{11}$ as subclasses of geo:SpatialThing.

Compound relationships between events can be modelled using the event:sub_event property. This can be used to capture the compound nature of disruptions: for example, a $t d$ :RoadClosure occurring as part of a $t d$ :RoadWorks event. The event:factor property links events to owl:Things that are factors of the event. This can capture additional relationships between events, such as a $t d$ :AbnormalTraffic event contributing to a $t d$ :Public TransportDelay event.

\section{Social Journeys Use Case}

A motivation for the Transport Disruption ontology is to support the use of integrated data sets of travel and transport information to identify disruptions that agents may encounter during travel. The Social Journeys project ${ }^{12}$ is developing a system to provide this information for bus users within the city of Aberdeen, UK. This section discusses the use of the Transport Disruption ontology within the project and outlines a versatile approach for obtaining the data required by the system from open data repositories ${ }^{13}$.

\subsection{Integrated Travel and Transport Data Sets}

The Social Journeys knowledge base (a sample of which is provided in Figure 2) contains several types of data that can be used to geolocate an event, reflecting

\footnotetext{
7 Namespace http://www.w3.org/2006/time\#, abbreviated to 'time'.

${ }^{8}$ Namespace http://purl.org/NET/c4dm/timeline.owl\#, abbreviated to 'tl'.

9 Namespace http://www.w3.org/2003/01/geo/wgs84_pos\#, abbreviated to 'geo'.

10 Namespace http://linkedgeodata.org/ontology/, abbreviated to 'lgd'.

11 Namespace http://vocab.org/transit/terms/, abbreviated to 'transit'.

12 http://www.dotrural.ac.uk/socialjourneys

13 Associated code is available at https://github.com/SocialJourneys.
} 


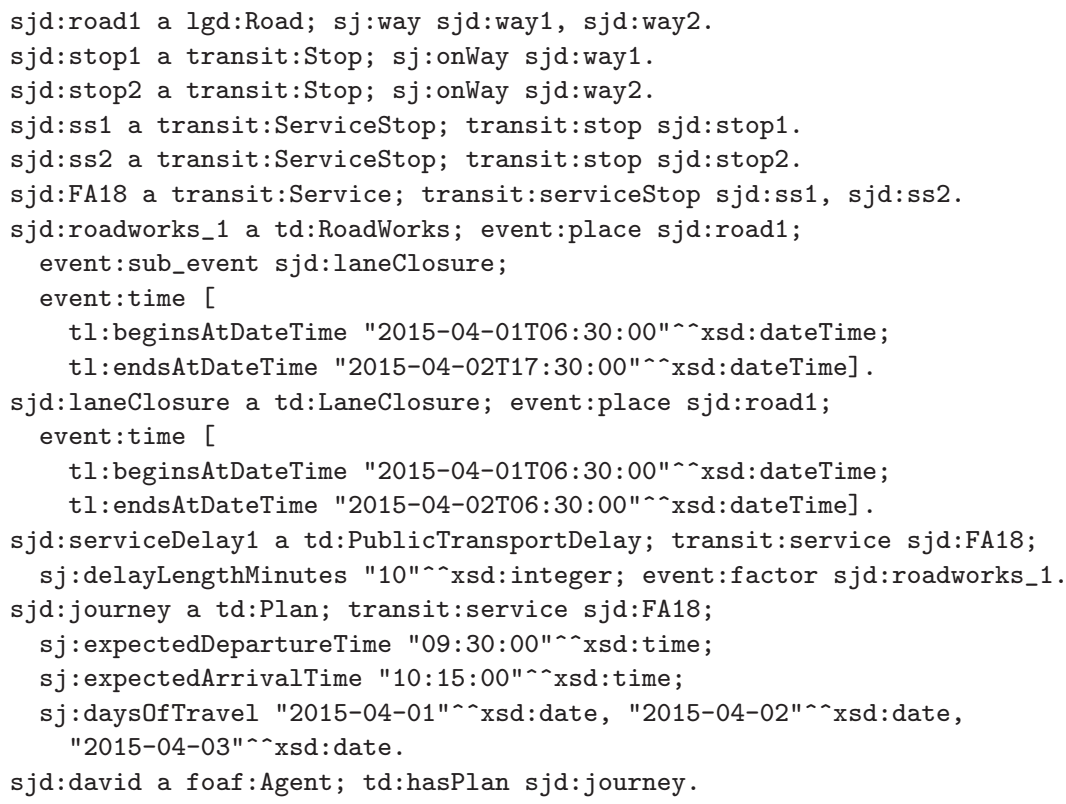

Fig. 2. Sample of the Social Journeys knowledge base.

the variety of location references used by providers of travel disruption information. This includes details of roads within Aberdeen, which are extracted ${ }^{14}$ from OpenStreetMap ${ }^{15}$ (OSM). The exported XML file consists of nodes, geospatial points with latitude and longitude values, and ways, ordered lists of nodes used to represent polygons (e.g. buildings) and polylines (e.g. roads) with attributes providing further details such as the building or road name. A script converts this into RDF and imports it to the Social Journeys knowledge base.

Access points to public transport and details of localities are used to associate events with bus stops, railway stations, airports and areas (localities) within the city. A triplication script converts the CSV data files for the NaPTAN and NPTG open data sets ${ }^{16}$ published by the UK Department for Transport ${ }^{17}$. Extracted bus stops are also linked to the description of the road that they are on.

Details of bus services within the city are extracted from the Traveline open data repository ${ }^{18}$ of bus operators, routes, and schedules. This data is available

$\overline{14 \text { Road details }}$ are extracted using the tool at http://extract.bbbike.org.

15 http://www.openstreetmap.org

16 NaPTAN provides details of public transport access points in the UK, (http:// data.gov.uk/dataset/naptan), and NPTG defines the areas of the UK served by public transport (http://data.gov.uk/dataset/nptg).

17 http://data.gov.uk/publisher/department-for-transport

18 http://www.travelinedata.org.uk/traveline-open-data 
as XML files encoded using the TransXChange schema ${ }^{19}$; a parser creates RDF descriptions using the Transit ontology of bus services that use stops within Aberdeen. The extracted bus service descriptions include the service name, operator, and references to the bus stops used.

Event descriptions are currently manually created based on reports from sources including the transport authority ${ }^{20}$, bus operator ${ }^{21}$, and a local radio station $^{22}$. Scripts to create event descriptions automatically are being developed.

Finally, as part of the system being developed, travellers are requested to provide details of their planned bus journeys. Each journey is described in terms of the departure and arrival times, days of travel, and the journey stages. Each stage defines the origin bus stop, bus service used, and destination stop.

\subsection{Identifying Potential Disruptive Impacts}

Potential travel disruptions can be identified by performing spatial and temporal matching of the events and planned journeys in the knowledge base. For example, a spatial match can be based on a common piece of infrastructure (e.g. road), while a temporal match can be determined if the time period of an event's occurance intersects with that of a journey, as illustrated by following query:

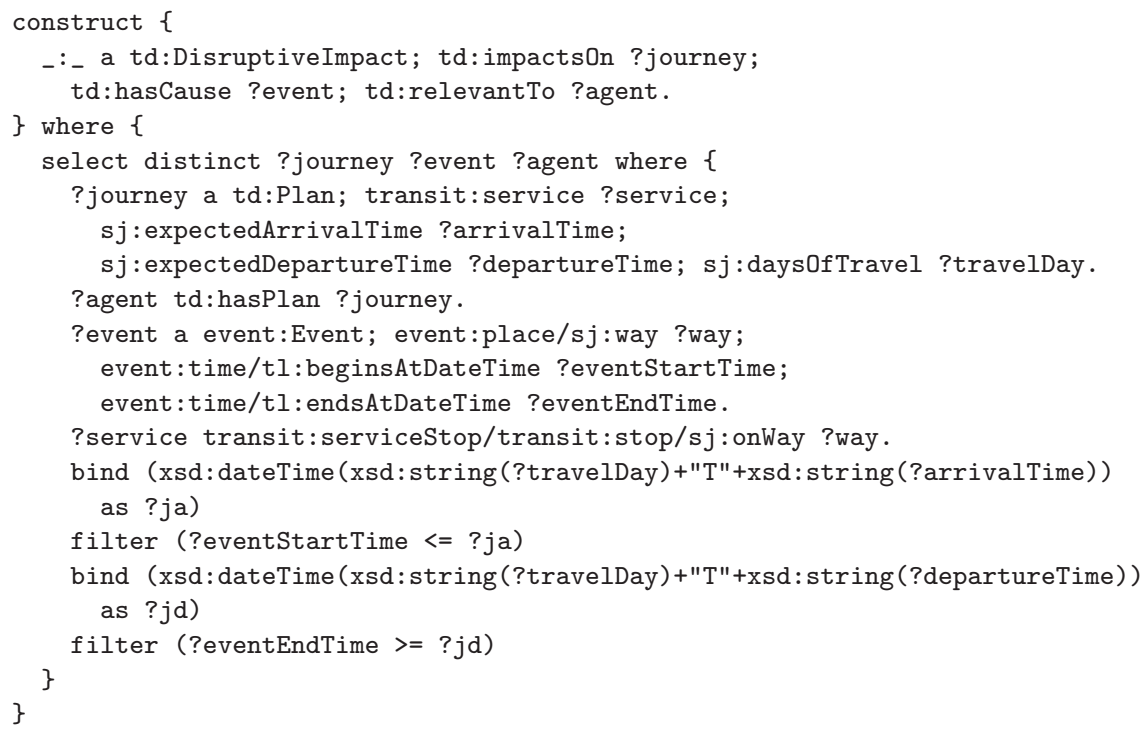

The td:DisruptiveImpact individuals returned by this query could be used by the system to alert users about the possible disruption. The simple approach shown to spatial matching could be extended to include more complex spatial

\footnotetext{
19 https://www.gov.uk/government/collections/transxchange

20 http://trafficscotland.org/

21 http://www.firstgroup.com/ukbus/aberdeen/travel_news/service_updates/

22 http://www. northsound1. com/travel
} 
reasoning, for example, using GeoSparq ${ }^{23}$ functions for boundary box matches. Storing the $t$ d:DisruptiveImpact individuals (after assigning a URI) would enable stakeholders to perform various types of analysis. For example, the bus operator could attempt to identify which bus services may be affected by events today or tomorrow, or, as part of reviewing historical performance, query for any services that were affected by disruptions during a specific time period.

This example illustrates the initial work necessary for intelligent travel disruption reporting. However, the knowledge base is by its nature dynamic, changing as futher details of events become available or new events occur. Future work will focus on recording the provenance of knowledge base updates and any reasoning performed with the data. This will involve alignment of the Transport Disrupion ontology with PROV-O ${ }^{24}$, the $\mathrm{W} 3 \mathrm{C}$ recommendation for representing provenance information. Indicating the quality, particularly of $t$ d:DisruptiveImpact and td:DisruptiveEvent individuals will be a key factor in supporting people decide if they should act upon the data or not. We plan to use the Qual-O ontology and assessment framework [1] to define metrics that evaluate the quality of such data. For example, a metric could rate the relevancy of a td:DisruptiveImpact to a person by determining if roadworks are located on their expected route of travel: if so the relevancy would be high, otherwise the relevancy score would be reduced based on the proximity of the roadworks to the route. This reflects the observation that while roadworks may be on the same road (as in the example above) they may not actually be encountered by the person.

\section{Related Work}

Along with semantic models of public transport routes and schedules provided by the Transit and GTFS ${ }^{25}$ ontologies, models have also been defined for other aspects of the transport domain. These include the Road Traffic Management Ontology ${ }^{26}$, the scope of which is limited to describing the actions a moving vehicle can perform (e.g. accelerate, change lane), and its relation to other vehicles (e.g. relative speed, road position). The draft Road Accident Ontology ${ }^{27}$ models road accidents in terms of the vehicles and living beings involved, relevant documents (e.g. driver licence, insurance certificate), location, and organisations (e.g. insurance companies). However, it is limited to only defining a single type of event (road accident) and does not consider any consequential impact. The Passim ontology ${ }^{28}$ models systems that convey transport information to travellers. Passim models such systems in terms of name, how it is accessed (website, SMS, mobile application), and coverage in terms of the modes of transport and towns, cities, and geographic regions that information is provided for.

\footnotetext{
23 http://geosparql.org

24 http://www.w3.org/ns/prov\#

25 http://vocab.gtfs.org/terms

26 http://lov.okfn.org/dataset/lov/vocabs/traffic

27 http://www.w3.org/2012/06/rao.html\#owl

28 http://data.lirmm.fr/ontologies/passim
} 


\section{Conclusion}

Ontologies provide a key technology for supporting data integration. Alignment of the Transport Disruption ontology with existing models, such as Event, FOAF, Transit, and LinkedGeoData extends the existing semantic modelling capabilities for integrated mobility data sets. The Transport Disruption ontology enables descriptions of travel and transport related events and their disruptive impacts on mobility. The defined event types and details of their impacts can be extended as necessary for use in different applications. As such, we argue that the Transport Disruption ontology provides a necessary component in enabling the contribution of Semantic Web efforts to addressing the mobility challenges faced by society today and in future smart cities. Along with the future work discussed above, we plan further evaluation of the ontology through use cases explored in collaboration with the Semantic Web and transport research communities.

\section{References}

1. C. Baillie, P. Edwards, and E. Pignotti. Qual: A provenance-aware quality model. Journal of Data and Information Quality, 5(3):12:1-12:22, Mar. 2015.

2. BSI Group. PAS 181:2014 Smart city framework - Guide to establishing strategies for smart cities and communities, 2014.

3. D. Corsar, P. Edwards, C. Baillie, M. Markovic, K. Papangelis, and J. Nelson. Short paper: Citizen sensing within a real time passenger information system. In Proceedings of the 6th International Workshop on Semantic Sensor Networks., volume 1063, pages 77-82. CEUR, 2013.

4. European Commission. ITS Roadmap Outline Intelligent Transport Systems for more efficient, safer, cleaner road transport, 2007.

5. B. Hyland, G. Atemezing, and B. Villazon-Terrazas. Best practices for publishing linked data. W3C Working Group Note, 2009. http://www.w3.org/TR/ld-bp/.

6. E. Jenelius and L.-G. Mattsson. Road network vulnerability analysis of areacovering disruptions: A grid-based approach with case study. Transportation Research Part A: Policy and Practice, 46(5):746 - 760, 2012. Network vulnerability in large-scale transport networks.

7. G. Marsden and I. Docherty. Insights on disruptions as opportunities for transport policy change. Transportation Research Part A: Policy and Practice, 51(0):46 $55,2013$.

8. MasterCard Enterprise Partnerships. Connecting cities Mobility: Unlocking potential in emerging markets, 2015.

9. T. Omitola, J. Breslin, and P. Barnaghi, editors. S4SC 2014 Semantics for Smarter Cities, volume 1280. CEUR Workshop Proceedings, 2015.

10. Passengerfocus. Bus passenger views on value for money, 2013

11. Passengerfocus. Passenger information when trains are disrupted, 2014.

12. B. Pender, G. Currie, A. Delbosc, and N. Shiwakoti. Improving bus bridging responses via satellite bus reserve locations. Journal of Transport Geography, 34(0):202 - 210, 2014.

13. The European Innovation Partnership on Smart Cities and Communities. Strategic implementation plan, 2013. 